\section{Catastrophe theory and brightness judgments}

\author{
THOMAS J. AYRES \\ Clarkson College of Technology, School of Management \\ Potsdam, New York, 13676
}

Human behavior is complex. As a result, psychologists are constantly searching for models and methods to simplify the analysis. One technique is to borrow a set of ideas from another discipline and graft them onto behavior. Initial enthusiasms and generalizations, however, must give way, after reflection, to more moderate claims, as with, for example, information theory and sociobiology.

A recent addition to this approach is catastrophe theory (Deakin, 1980; Isnard \& Zeeman, 1974). This outgrowth of topology describes the form of response surfaces containing discontinuities in terms of a set of controlling dimensions. The attraction of this difficult branch of applied mathematics is its alleged potential for dealing with complex behaviors. As such, it should be used only when simpler, more parsimonious explanations are unavailable.

A case in point is the fitting of a catastrophe model to brightness judgments (Yelen, 1980). The method of limits was used to collect brightness-discrimination data for a bright and a dim standard (110 and $31 \mathrm{~V}$, respectively, to a $7.5-\mathrm{W}$ bulb). In a descending series, for example, the standard was presented simultaneously with a more luminous variable stimulus, and the subject reported whether or not the variable seemed brighter than the standard. Luminance of the variable was then reduced on each succeeding trial. Hysteresis-the tendency to keep on saying "yes" in a descending series or "no" in an ascending series, past the point of subjective equality (e.g., Woodworth \& Schlosberg, 1954)-was observed only for the series with the bright standard. Thus, the results appeared to conform to a "cusp" catastrophe, in which the progression from "yes" to "no" responses on a surface folded in the third dimension was controlled by the $\Delta \mathrm{I}$ dimension, while the size of the hysteresis effect was a function of the brightness of the standard.

The puzzling aspect of this application, aside from the fact that a single variable (brightness) has been used on two dimensions, is that change of brightness is plotted in volts (steps of $2.4 \mathrm{~V}$ ). Perhaps it would be more psychologically appropriate to consider the data in terms of a percent-change of luminance under the simple assumption that $\Delta I / I$ for equal sensation is roughly constant over the range studied. Such a translation of the results is shown in Figure 1. Inspection shows that hysteresis occurred for both the bright

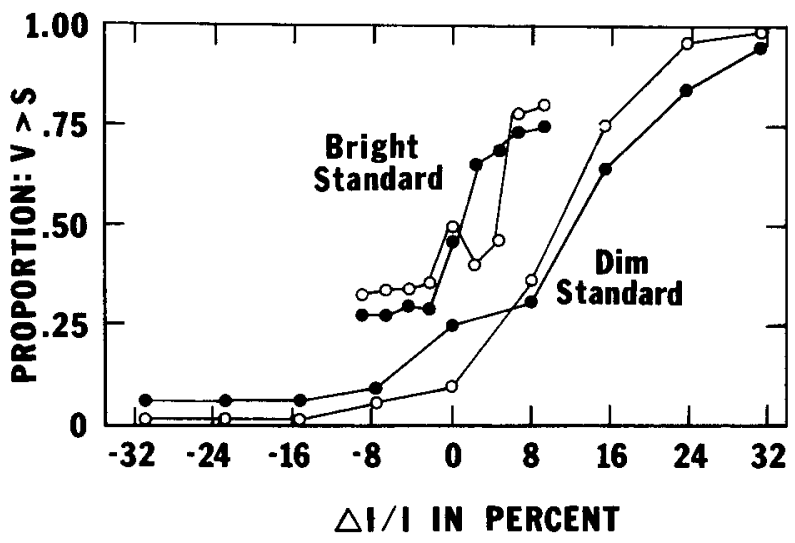

Figure 1. The proportion of judgments that the variable was brighter than the standard as a function of the percent-change in intensity of the variable relative to the standard. Ascending series are shown by open circles, and descending series are shown by filled circles. Data were read from Figure 1 of Yelen (1980).

and the dim standard. With the dim standard, however, the use of subjectively large step sizes confined the hysteresis to a single step in the series, thus apparently eliminating the effect. Nevertheless, there is no evidence to support the claim that hysteresis was greater for one series than for the other.

It is still possible to point out that each condition is an example of a simple "fold" catastrophe, in which a single control dimension $-\Delta \mathrm{I}-$ governs a response function with a bivalued region. Such an assertion, however, does not really add much to our understanding of the situation; the terms "hysteresis" and "habituation error" are equally useful. This is not to say that the behavior of the subjects is entirely explained in this way-for example, why do the responses for the bright standard cover a smaller range of certainty than do the other responses? The problem is that, in this instance, catastrophe theory does not help us with the basic questions of psychophysics.

\section{REFERENCES}

Deakin, M. A. B. Applied catastrophe theory in the social and biological sciences. Bulletin of Mathematical Biology, 1980, 42, 647-679.

ISNARD, C. A., \& ZEeman, E. C. Some models from catastrophe theory in the social sciences. In L. Collins (Ed.), Use of models in the social sciences. London: Tavistock, 1974.

WoOdWorth, R. S., \& SchlosBerg, H. Experimental psychology. New York: Holt, 1954.

YELEN, D. R. A catastrophe model for the effects of a response set on a discrimination task. Perception \& Psychophysics, 1980, 28, 177-178.

(Received for publication February 9, 1981; accepted February 16, 1981.) 